\title{
ON SYMMETRIZABILITY OF HYPERBOLIC MATRIX SPACES
}

\author{
E. YU. PANOV
}

\begin{abstract}
A new symmetrizability criterion for linear matrix spaces is proposed, with applications to the theory of first order conservation laws.
\end{abstract}

Let $L \subset \operatorname{Mat}(n, k)$ be a real linear subspace of the space of $(n \times n)$-matrices with coefficients from the field $k=\mathbb{R}$ or $\mathbb{C}$.

Definition 1. The family $L$ is said to be hyperbolic if

$$
A^{2} \in L \text { for all } A \in L,
$$

and all matrices in $L$ have a simple real spectrum (i.e., the eigenvalues of any matrix $A \in L$ are real and there is a basis consisting of the corresponding eigenvectors).

Condition (11) implies the relation

$$
A B+B A=(A+B)^{2}-A^{2}-B^{2} \in L \quad \text { for all } A, B \in L
$$

(this means that $L$ is a special Jordan algebra). In particular, we can define linear operators $S_{A}$ on $L$ by the rule $S_{A} B=A B+B A$.

If $L$ is a hyperbolic space, then its extension $\{A+\lambda E \mid \lambda \in \mathbb{R}\}$, obtained by adding the unit matrix $E$, is also a hyperbolic space.

The hyperbolicity condition admits the following reformulation.

Proposition 1. A space $L$ that satisfies (11) and contains the unit matrix $E$ is hyperbolic if and only if the linear operators $S_{A}$ have a simple real spectrum in $L$ for all $A \in L$.

Proof. Let $L$ be a hyperbolic space. Then the spectrum $\sigma(A)$ of every matrix $A \in L$ is simple and real. We consider the symmetric bilinear form $(A, B)=\operatorname{Tr} A B$. Then $(A, A)=\sum_{\lambda \in \sigma(A)} \lambda^{2}>0$ for $A \neq 0$. Therefore, the form $(\cdot, \cdot)$ is positive definite and determines a scalar multiplication on $L$. Direct verification shows that the operators $S_{A}$ are symmetric with respect to this scalar multiplication (i.e., $\left(S_{A} B, C\right)=\left(B, S_{A} C\right)$ for all $B, C \in L)$; consequently, they have simple real spectra.

Conversely, suppose that each operator $S_{A}$ has a simple real spectrum in $L$. Relation (11) and the condition $E \in L$ imply that $L$ contains all powers $A^{n}, n \geq 0$, for $A \in L$, so that $f(A) \in L$ for every real polynomial $f(z)$. Let $A \in L$. By our assumptions, the operator $S_{A}$ has a simple real spectrum. Clearly, the simplicity of the spectrum of a matrix (or an operator) $A$ means that there exists a polynomial $p(z)=\prod_{k=1}^{m}\left(z-\lambda_{k}\right)$ with distinct real roots $\lambda_{k}, k=1, \ldots, m$, such that $p(A)=0$. Therefore, there exists a polynomial $p(z)$ with distinct real roots such that $p\left(S_{A}\right)=0$. Since $p(2 A)=p\left(S_{A}\right) E=0$ and the polynomial $p(2 z)$ also has distinct real roots, the spectrum of $A$ is real and simple. The proof is complete.

2000 Mathematics Subject Classification. Primary 15A30, 15A06.

Key words and phrases. Hyperbolic matrix space, spectrum, symmetrizable system.

Supported by RFBR (grant no. 06-01-00289) and by Deutsche Forschungsgemeinschaft (DFG project no. 436 RUS 113/895/0-1). 
We note that the assumption $E \in L$ is essential for the inverse statement of Proposition 1. Indeed, let $J$ be any nontrivial matrix such that $J^{2}=0$ and $L=\{\lambda J \mid \lambda \in \mathbb{R}\}$. Obviously, $L$ satisfies (11), but $E \notin L$. It is easily seen that $S_{A}=0$ for all $A \in L$, but $L$ is certainly not a hyperbolic space.

As follows from Proposition 1, the hyperbolicity condition means that the system of conservation laws corresponding to the Burgers-like equation $U_{t}+\left(U^{2}\right)_{x}=0, U=$ $U(t, x) \in L$ is hyperbolic. In the papers [3, 4, general systems of the form

$$
U_{t}+f(U)_{x}=0
$$

were studied, in which the unknown function $U=U(t, x)$ takes its values in the space $S_{n}$ of symmetric matrices of order $n$ or in the space $H_{n}$ of Hermitian matrices of order $n$, and $U \rightarrow f(U)$ is the functional calculus operator. In those papers it was shown that system (2) is hyperbolic. More generally, systems such as (2) can also be considered in the case where $U$ takes its values in an arbitrary matrix linear space $L$ invariant under the functional calculus operators: $f(U) \in L$ for all $U \in L$ and all real functions $f(u)$ admitting an analytic extension to the entire complex plane. In particular, the space $L$ must satisfy (11). Our Theorem 1 (see below) shows that for a nonlinear function $f$, system (2) is hyperbolic only in the case, studied in [3, 4, where $L$ consists of symmetric or Hermitian matrices (after an appropriate choice of a basis in $k^{n}$ ).

Before formulating our main result, we describe some useful constructions preserving the property of hyperbolicity.

Let $L$ be a matrix space. We introduce the space $L^{*}$ consisting of all conjugate matrices $A^{*}$ with $A \in L$ (with respect to some scalar multiplication on $k^{n}$ ). Clearly, the space $L^{*}$ is hyperbolic simultaneously with $L$. Now, suppose that $H \subset k^{n}$ is a linear subspace invariant under the action of $L$, i.e., $A(H) \subset H$ for all $A \in L$. Then, we can define matrix spaces $L_{H}$ and $L_{/ H}$ to consist of all matrices corresponding to the restricted operators $\left.A\right|_{H}: H \rightarrow H, A \in L$, and to the factor operators $A / H: k^{n} / H \rightarrow k^{n} / H, A \in L$, respectively. Obviously, the orthogonal complement $H^{\perp}$ is an invariant space for $L^{*}$ and $\left(L_{/ H}\right)^{*}=\left.L^{*}\right|_{H^{\perp}}$. The following simple statement is true.

Lemma 1. Suppose $L$ is a hyperbolic matrix space and a subspace $H \subset k^{n}$ is invariant under the action of $L$. Then the matrix spaces $L_{H}$ and $L_{/ H}$ are hyperbolic.

Proof. By the duality relation $L_{/ H}=\left(\left.L^{*}\right|_{H^{\perp}}\right)^{*}$, it suffices to prove the lemma in the case of the matrix space $L_{H}$. Clearly, $L_{H}$ satisfies (1). In the proof of Proposition 1 it was shown that for any matrix $A \in L$ there exists a polynomial $p(z)$ with distinct real roots such that $p(A)=0$. Then $p\left(\left.A\right|_{H}\right)=\left.p(A)\right|_{H}=0$. Therefore, the spectrum of $\left.A\right|_{H}$ is real and simple. Hence, the space $L_{H}$ is hyperbolic, as required.

We are ready to formulate the main result.

Theorem 1. A space $L$ satisfying (1) is hyperbolic if and only if all matrices $A \in L$ are symmetric (Hermitian) with respect to some scalar multiplication in $k^{n}:(A u, v)=(u, A v)$ for all $u, v \in k^{n}$.

Below it is assumed that $L$ is a space of matrices over the field $k=\mathbb{C}$. The case of the real field $k=\mathbb{R}$ reduces to that of $k=\mathbb{C}$ by complexification. Indeed, if a real matrix family $L$ consists of Hermitian matrices with respect to a scalar multiplication $(\cdot, \cdot)$ on $\mathbb{C}^{n}$, then the matrices in $L$ are symmetric with respect to the real scalar multiplication $\operatorname{Re}(\cdot, \cdot)$ on $\mathbb{R}^{n}$. Observe that $L$ remains hyperbolic after complexification.

To prove Theorem 1, we need some preliminary results and constructions. 
Lemma 2. For all $A, B, C \in L$ we have

$$
\left[S_{A}, S_{B}\right] C=[[A, B], C] .
$$

Here $[\cdot, \cdot]$ is the commutator of operators (matrices).

Proof. The claim follows directly from the identity

$$
\begin{aligned}
{\left[S_{A}, S_{B}\right] C } & =S_{A} S_{B} C-S_{B} S_{A} C \\
& =A B C+A C B+B C A+C B A-B A C-B C A-A C B-C A B \\
& =(A B-B A) C-C(A B-B A)=[[A, B], C] .
\end{aligned}
$$

Corollary 1. 1) $[[A, B], C] \in L$ for all $A, B, C \in L$. 2) Let $[L, L]$ be the linear hull of the set of commutators $[A, B], A, B \in L$. Then $[L, L]$ is a Lie algebra (with the multiplication $[\cdot, \cdot])$.

Proof. Statement 1) readily follows from Lemma 2. To prove 2), it suffices to verify that $\left[\left[A_{1}, B_{1}\right],\left[A_{2}, B_{2}\right]\right] \in[L, L]$ for all $A_{1}, B_{1}, A_{2}, B_{2} \in L$. But this is implied by the identity

$$
\left[\left[A_{1}, B_{1}\right],\left[A_{2}, B_{2}\right]\right]=\left[\left[\left[A_{1}, B_{1}\right], A_{2}\right], B_{2}\right]-\left[\left[\left[A_{1}, B_{1}\right], B_{2}\right], A_{2}\right]=\left[C_{1}, B_{2}\right]-\left[C_{2}, A_{2}\right],
$$

where $C_{1}=\left[\left[A_{1}, B_{1}\right], A_{2}\right], C_{2}=\left[\left[A_{1}, B_{1}\right], B_{2}\right] \in L$; see statement 1$)$.

We consider the linear subspace $\mathcal{A}=[L, L] \oplus L$ and define a multiplication on $\mathcal{A}$ by setting, for $x=X \oplus A, y=Y \oplus B$,

$$
x y=([X, Y]-[A, B]) \oplus([X, B]-[Y, A]) .
$$

Observe that, by Corollary $1,[X, Y],[A, B] \in[L, L]$ and $[X, B],[Y, A] \in L$ for any $X$, $Y \in[L, L]$ and any $A, B \in L$, so that this multiplication is well defined.

We define the subspaces

$$
\begin{aligned}
& Z_{1}=\{X \in[L, L] \mid[X, B]=0 \text { for all } B \in L\}, \\
& Z_{2}=\{A \in L \mid[A, B]=0 \text { for all } B \in L\}
\end{aligned}
$$

of the spaces $[L, L]$ and $L$, respectively.

Lemma 3. 1) $\mathcal{A}$ is a Lie algebra, and its center $Z(\mathcal{A})$ coincides with $\left.Z_{1} \oplus Z_{2} ; 2\right)$ the maps

$$
f(X \oplus A) B=[X, B]+i S_{A} B, \quad h(X \oplus A) v=X v+i A v
$$

are linear representations of $\mathcal{A}$ in the spaces $L \otimes \mathbb{C}$ and $\mathbb{C}^{n}$, respectively. Here $i^{2}=-1$.

Proof. 1) The fact that $\mathcal{A}$ is a Lie algebra is verified directly with the help of the known properties of commutators. We omit the corresponding boring calculations. To describe the center $Z(\mathcal{A})$, suppose that $x=X \oplus A \in Z(\mathcal{A})$. Then $x y=0$ for all $y \in \mathcal{A}$. Taking $y=0 \oplus B$, we see that $[X, B]=[A, B]=0$ for all $B \in L$. Therefore, $x \in Z_{1} \oplus Z_{2}$. Conversely, if $x=X \oplus A \in Z_{1} \oplus Z_{2}$, then $X$ and $A$ commute with all matrices in $L$, and therefore, they also commute with the matrices in $[L, L]:[X, Y]=[A, Y]=0$ for all $Y \in[L, L]$ (this follows easily from the Jacobi identity). Now, (3) implies that $x y=0$ for all $y \in \mathcal{A}$, i.e., $x \in Z(\mathcal{A})$.

2) For $X \in[L, L]$, we define an operator $C_{X}$ acting in $L \otimes \mathbb{C}$ by the rule $C_{X} B=[X, B]$. Then $\left[C_{X}, C_{Y}\right]=C_{[X, Y]}$ and $\left[C_{X}, S_{A}\right]=S_{[X, A]}$ for $X, Y \in[L, L], A \in L$. Let $x=X \oplus A$, and let $y=Y \oplus B \in \mathcal{A}$. Then, using the above relations and Lemma 2, we obtain

$$
\begin{aligned}
{[f(x), f(y)] } & =\left[C_{X}+i S_{A}, C_{Y}+i S_{B}\right] \\
& =\left[C_{X}, C_{Y}\right]-\left[S_{A}, S_{B}\right]+i\left(\left[C_{X}, S_{B}\right]-\left[C_{Y}, S_{A}\right]\right) \\
& =C_{[X, Y]-[A, B]}+i S_{[X, B]-[Y, A]}=f(x y),
\end{aligned}
$$


and the map $f$ is a homomorphism of the Lie algebra $\mathcal{A}$ into the algebra $g l(L \otimes \mathbb{C})$ of linear operators in $L \otimes \mathbb{C}$, i.e., it is a linear representation of $\mathcal{A}$ in $L \otimes \mathbb{C}$. Next,

$$
[h(x), h(y)]=[X+i A, Y+i B]=[X, Y]-[A, B]+i([X, B]-[Y, A])=h(x y) ;
$$

i.e., $h$ is a representation of the algebra $\mathcal{A}$ in $\mathbb{C}^{n}$. The proof is complete.

First, we prove Theorem 1 in the case where $Z_{1}=\{0\}$.

Proposition 2. Suppose $L$ is a hyperbolic matrix space and $Z_{1}=\{0\}$. Then all matrices in $L$ are Hermitian with respect to some scalar multiplication in $\mathbb{C}^{n}$.

Proof. If $L$ is a hyperbolic matrix space, then its extension $\{A+\lambda E \mid \lambda \in \mathbb{R}\}$ obtained by adding the unit matrix $E$ is also a hyperbolic space with the same algebra $[L, L]$. Therefore, there is no loss of generality in assuming that $E \in L$. It is easily seen that the operators $f(x)$ are skew-Hermitian in $L \otimes \mathbb{C}$ with respect to the scalar product $(A, B)=\operatorname{Tr} A \bar{B}$, where $B \rightarrow \bar{B}$ denotes complex conjugation on $L \otimes \mathbb{C}$. Therefore, the symmetric bilinear form $(x, y)=-\operatorname{Tr} f(x) f(y)$ is nonnegative definite. Moreover, if $x=X \oplus A \in \mathcal{A}$ and $(x, x)=0$, then $f(x)=0$. In particular, $A=-\frac{i}{2} f(x) E=0$. Then $f(x) B=[X, B]=0$ for all $B \in L$, i.e., $X \in Z_{1}$. Since $Z_{1}=\{0\}$ by our assumptions, we have $X=0$. Hence, $x=0$, so that the form $(\cdot, \cdot)$ is nondegenerate. Therefore, this form determines a scalar product on $\mathcal{A}$. The operators $a d_{x} y=x y$ are skew-symmetric with respect to this scalar product. Indeed,

$$
\begin{aligned}
\left(a d_{x} y, z\right) & =-\operatorname{Tr} f(x y) f(z)=-\operatorname{Tr}[f(x), f(y)] f(z) \\
& =\operatorname{Tr} f(y)[f(x), f(z)]=\operatorname{Tr} f(y) f(x z)=-\left(y, a d_{x} z\right) .
\end{aligned}
$$

The above property means that $\mathcal{A}$ is a compact Lie algebra (in the sense of [1, 2]). By the known properties of compact Lie algebras (see, e.g., [1, 2]), we have $\mathcal{A}=\mathcal{A}_{1} \oplus Z(\mathcal{A})$, where $\mathcal{A}_{1}$ is a semisimple compact Lie algebra, which is the Lie algebra of a unique simply connected compact Lie group $G$. Moreover, the homomorphism $h: \mathcal{A}_{1} \rightarrow g l\left(\mathbb{C}^{n}\right)$ induces a homomorphism of Lie groups $\tilde{h}: G \rightarrow \operatorname{GL}\left(\mathbb{C}^{n}\right)$. Here $\operatorname{GL}\left(\mathbb{C}^{n}\right)$ is the Lie group of nonsingular linear operators on $\mathbb{C}^{n}$ with the corresponding Lie algebra $g l\left(\mathbb{C}^{n}\right)$. In other words, $G$ acts linearly on $\mathbb{C}^{n}: g v=\tilde{h}(g) v$. We decompose the space $\mathbb{C}^{n}$ into a direct sum of indecomposable subspaces invariant under the action of $L: \mathbb{C}^{n}=\bigoplus_{k=1}^{m} V_{k}$. If $x \in Z(\mathcal{A})$, then, by Lemma 3 and the condition $Z_{1}=0$, we have $x=0 \oplus A$, where $[A, B]=0$ for all $B \in L$. This implies that $A$ acts trivially on the spaces $V_{k}: A=\lambda_{k} E$ on $V_{k}$. Indeed, otherwise $V_{k}$ can be decomposed into a direct sum of proper subspaces that correspond to different eigenvalues of $A$ (recall that the restriction $\left.A\right|_{V_{k}}$ has a simple real spectrum, see Lemma 1 ), and these subspaces are invariant for all matrices $B \in L$, by the condition $[A, B]=0$ for all $B \in L$. But this contradicts the fact that $V_{k}$ is indecomposable. Clearly, all the subspaces $V_{k}, k=1, \ldots, m$, are invariant subspaces for the matrices in $\mathcal{A}$; consequently, they are invariant under the action of the group $G$.

We may assume that the scalar product in $\mathbb{C}^{n}$ is chosen in such a way that the spaces $V_{k}, k=1, \ldots, m$, are pairwise orthogonal. We define a new scalar product $(u, v)_{i}$ in $\mathbb{C}^{n}$, invariant under the action of $G$, by setting $(u, v)_{i}=\int_{G}(g u, g v) d \mu(g)$, where $\mu$ is the Haar measure in $G$. Relative to this scalar product, $\tilde{h}$ takes values in the group $U(n)$ of unitary operators (matrices), and consequently, for $x \in \mathcal{A}_{1}$ the image $h(x)$ is contained in the corresponding Lie algebra $u(n)$ of skew-Hermitian matrices. It is easily seen that the spaces $V_{k}, k=1, \ldots, m$, remain pairwise orthogonal under the new scalar product. Therefore, the matrices $h(x)$ are also skew-Hermitian for $x=0 \oplus A \in Z(\mathcal{A})$, because $h(x)=i A=i \lambda_{k} E$ on the subspaces $V_{k}$, and $\lambda_{k} \in \mathbb{R}, k=1, \ldots, m$. Thus, the image $h(\mathcal{A})$ belongs to $u(n)$, and since $h(0 \oplus A)=i A$, we see that all matrices $A \in L$ are Hermitian. The proof is complete. 
Now we prove that, in fact, our assumption $Z_{1}=0$ is always fulfilled.

Proposition 3. Let $L$ be a hyperbolic matrix space. Then $Z_{1}=\{0\}$.

Proof. We use induction on the dimension $n$. If $n=0$ or 1 , then $[L, L]=\{0\}$ and there is nothing to prove. Now, suppose that $n>1$ and that our statement is true for all dimensions less than $n$. Suppose $X \in[L, L]$ and $[X, B]=0$ for all $B \in L$. We need to check that $X=0$. Let $\mu \in \mathbb{C}$ be an eigenvalue of $X$, and let $H \subset \mathbb{C}^{n}$ be the corresponding subspace of eigenvectors. If $H=\mathbb{C}^{n}$, then $X=\mu E=0$ (this follows from the obvious relation $\operatorname{Tr} X=0)$, as required.

It remains to consider the case where $H$ is a proper subspace of $\mathbb{C}^{n}$. Since $X A v=$ $A X v=\mu A v$ for all $v \in H, A \in L$, we see that $H$ is invariant under the action of $L$ and, with it, of $[L, L]$. Therefore, we can define the homomorphisms of restriction $\left.A \rightarrow A\right|_{H}$ of the spaces $L$ and $[L, L]$ into the spaces $L_{H}$ and $\left[L_{H}, L_{H}\right]$, respectively. By Lemma 1 , $L_{H}$ is a hyperbolic space of order $m=\operatorname{dim} H<n$, and the matrix $\left.X\right|_{H}$ commutes with $L_{H}$. By the inductive hypothesis, we have $\left.X\right|_{H}=0$, that is, $\mu=0$ and $H=\operatorname{Ker} X$. If $V \subset \mathbb{C}^{n}$ is a proper linear subspace invariant under the action of $L$, then $V$ is also invariant under the action of $[L, L]$ and $\left.X\right|_{V} \in\left[L_{V}, L_{V}\right],\left[\left.X\right|_{V},\left.A\right|_{V}\right]=0$ for all $A \in L$. Again by the inductive hypothesis, we have $\left.X\right|_{V}=0$, i.e., $V \subset H$. Thus, $H$ contains all proper invariant subspaces.

Now, observe that $H_{1}=\operatorname{Im} X$ is an invariant subspace, which follows directly from the relation $A X v=X A v$ for all $A \in L, v \in \mathbb{C}^{n}$. Since $H_{1} \neq \mathbb{C}^{n}$ (otherwise $H=\operatorname{ker} X=\{0\}$, which is not true), we see that $H_{1}$ is a proper invariant subspace, whence $H_{1} \subset H$, i.e., $X^{2}=0$. As shown above, $L_{H}$ is a hyperbolic space for which $Z_{1}=0$. By Proposition 2, there exists a scalar multiplication on $H$ under which the matrices $\left.A\right|_{H}$ are Hermitian for all $A \in L$. Let $H_{2}=H \ominus H_{1}$ be the orthogonal complement to $H_{1}$ in $H$. Since the matrices $A \in L$ are Hermitian on $H$, if follows that $H_{2}$ is invariant under the actions of $L$ and $[L, L]$. Hence, we can consider the space $L_{/ H_{2}}$, which is hyperbolic by Lemma 1 . It is clear that $X / H_{2}$ belongs to $\left[L_{/ H_{2}}, L_{/ H_{2}}\right]$ and commutes with $L_{/ H_{2}}$. Assume that $H_{2} \neq\{0\}$, i.e., $\operatorname{dim} \mathbb{C}^{n} / H_{2}<n$. Then, by the inductive hypothesis, $X / H_{2}=0$. But this contradicts the fact that $\operatorname{Im} X / H_{2}=H / H_{2} \simeq H_{1} \neq\{0\}$. Thus, $H_{2}=0$, i.e., $H_{1}=H$. This implies that $\mathbb{C}^{n}=\left(\mathbb{C}^{n} / H\right) \oplus H$ and the operator $X$ gives rise to an isomorphism $X: \mathbb{C}^{n} / H \rightarrow H$. Identifying $\mathbb{C}^{n} / H$ and $H$ via this isomorphism, we see that $\mathbb{C}^{n}=H \oplus H$ and $X(u, v)=(0, u)$. Any operator $A \in L$ can be represented in the form $A(u, v)=\left(A_{1} u, A_{2} u+A_{3} v\right)$, because the space $0 \oplus H$ is invariant. Since $0=[X, A](u, v)=\left(0, A_{1} u-A_{3} u\right)$, we have $A_{3}=A_{1}$. Next, if $A, B \in L$ and $A(u, v)=$ $\left(A_{1} u, A_{2} u+A_{1} v\right), B(u, v)=\left(B_{1} u, B_{2} u+B_{1} v\right)$, then a direct computation shows that $[A, B](u, v)=\left(C_{1} u, C_{2} u+C_{1} v\right)$, where $C_{1}=\left[A_{1}, B_{1}\right], C_{2}=\left[A_{2}, B_{1}\right]+\left[A_{1}, B_{2}\right]$. In particular, $\operatorname{Tr} C_{1}=\operatorname{Tr} C_{2}=0$. Clearly, this property holds true for all matrices in $[L, L]$, because they are linear combinations of commutators $[A, B], A, B \in L$. Since $X \in[L, L]$ and $X(u, v)=(0, E u)$, where $E$ is the unit matrix, we obtain the wrong relation $\operatorname{Tr} E=0$. This contradiction shows that $X=0$. The proof is complete.

We are ready to finish the proof of Theorem 1 . The direct statement of Theorem 1 immediately follows from Propositions 2 and 3. Conversely, if all matrices in a linear matrix space $L$ are Hermitian, then they have simple real spectra, and consequently, the space $L$ is hyperbolic. The proof of the theorem is complete.

Now, we apply our result to the problem of symmetrizability for the first order system

$$
u_{t}+\sum_{k=1}^{m} A_{k} u_{x_{k}}=0, \quad A_{k}=A_{k}(t, x, u) \in \operatorname{Mat}(n, \mathbb{R}), k=1, \ldots, m .
$$


Recall that system (4) is symmetrizable if, for fixed $t, x, u$, all matrices $A_{k}, k=1, \ldots, m$, can be symmetrized simultaneously by an appropriate choice of a basis or, equivalently, by the choice of a scalar product $(B u, v)$ given by some positive definite matrix $B$. Multiplying system (4) by the matrix $B$, we arrive at the following symmetric form of that system:

$$
B u_{t}+\sum_{k=1}^{m} C_{k} u_{x_{k}}=0
$$

where the matrices $B$ and $C_{k}, k=1, \ldots, m$, are symmetric and $B$ is positive definite.

The symmetrizability of system (4) can be stated as the capability of symmetrizing all matrices in the real linear hull $M$ of the matrices $A_{k}, k=1, \ldots, m$. Clearly, the hyperbolicity condition

$A$ has a simple real spectrum for all $A \in M$

is necessary for the symmetrizability of the real linear matrix subspace $M \subset \operatorname{Mat}(n, \mathbb{C})$. In the case of complex matrices, symmetrizability is understood as being able to reduce all matrices in $M$ to Hermitian form. In the cases where $m=1$ or $n=2$, condition (5) is also sufficient for symmetrizability (see, e.g., [5]).

It turns out that this remains true only in the cases indicated. If $n>2$, then condition (5) and even the stronger condition of strict hyperbolicity,

$$
A \text { has distinct and real eigenvalues for all } A \in M, A \neq 0,
$$

does not suffice for the symmetrizability of a matrix space $M$ with $\operatorname{dim} M>1$. The corresponding example was constructed in [5]. For completeness, we present this example below.

Example. For $n=3$, consider the two-dimensional linear matrix space $M$ that consists of matrices of the form

$$
A=\left(\begin{array}{ccc}
0 & 0 & a-b \\
0 & 0 & b \\
a-b & a & 0
\end{array}\right), \quad a, b \in \mathbb{R} .
$$

The eigenvalues of $A$ are computed easily: $\lambda_{1}=0, \lambda_{2,3}= \pm \sqrt{(a-b)^{2}+a b}$. They are real and distinct for $A \neq 0$, because the quadratic form $(a-b)^{2}+a b$ is positive definite. Thus, condition (6) is satisfied. We prove that this "strictly hyperbolic" family cannot be symmetrized. Assuming the contrary, we find a scalar product $(P x, y)$ corresponding to some positive definite matrix

$$
P=\left(\begin{array}{lll}
p_{1} & p_{2} & p_{3} \\
p_{2} & p_{4} & p_{5} \\
p_{3} & p_{5} & p_{6}
\end{array}\right)
$$

such that all matrices $A \in M$ are symmetric. Then $(P A x, y)=(x, P A y)$; i.e., the matrices $P A$ are symmetric under the original scalar product. Writing the relations $(P A)_{12}=(P A)_{21},(P A)_{13}=(P A)_{31}$, and $(P A)_{23}=(P A)_{32}$ explicitly, we see that $a p_{3}=(a-b) p_{5},(a-b) p_{1}+b p_{2}=(a-b) p_{6},(a-b) p_{2}+b p_{4}=a p_{6}$ for all $a, b \in \mathbb{R}$. This implies that $p_{i}=0, i=1, \ldots, 6$, i.e., $P=0$. But this contradicts the condition $P>0$. Therefore, the family $M$ is not symmetrizable. Taking the basis matrices $A_{1}, A_{2}$ corresponding to $a=1, b=0$, and $a=b=1$, we arrive at a strictly hyperbolic but not symmetrizable system $q_{t}=A_{1} q_{x}+A_{2} q_{y}=0, q=(u, v, w)^{\top}$ of the form

$$
\left\{\begin{array}{l}
u_{t}=w_{x} \\
v_{t}=w_{y} \\
w_{t}=(u+v)_{x}+v_{y} .
\end{array}\right.
$$


One criterion for symmetrizability was found in [5], saying that a space $M$ can be symmetrized if and only if all matrices in the minimal real Lie algebra containing $i A$, $A \in M$ (with $i^{2}=-1$ ), have a simple imaginary spectrum.

Now we are able to introduce a new symmetrizability criterion, which is an easy consequence of Theorem 1 . We denote by $L=L(M)$ the minimal linear matrix subspace that contains $M$ and satisfies condition (1).

Theorem 2. The family $M$ is symmetrizable if and only if the space $L$ is hyperbolic.

Proof. If all matrices $A \in M$ are symmetric (Hermitian) under some scalar product, then the same is true for the matrices in $L$. Consequently, each of them has a simple real spectrum; i.e., the space $L$ is hyperbolic.

The converse statement follows directly from Theorem 1.

Observe that the result on nonsymmetrizability in the above example follows from Theorem 2. Indeed, let

$$
A_{1}=\left(\begin{array}{ccc}
0 & 0 & 1 \\
0 & 0 & 0 \\
1 & 1 & 0
\end{array}\right), \quad A_{2}=\left(\begin{array}{lll}
0 & 0 & 0 \\
0 & 0 & 1 \\
0 & 1 & 0
\end{array}\right)
$$

be the basis matrices defined in this example. Then the matrix

$$
\left(\begin{array}{lll}
0 & 0 & 0 \\
0 & 0 & 1 \\
0 & 0 & 0
\end{array}\right)=A_{2} A_{1} A_{2}=\frac{1}{2}\left[\left(S_{A_{2}}\right)^{2} A_{1}-S_{A_{2}^{2}} A_{1}\right]
$$

belongs to $L$, but its spectrum is not simple.

\section{REFERENCES}

[1] M. Goto and F. D. Grosshans, Semisimple Lie algebras, Lecture Notes in Pure and Appl. Math., vol. 38, Marcel Dekker, Inc., New York-Basel, 1978. MR0573070 (58:28084)

[2] J.-P. Serre, Lie algebras and Lie groups, W. A. Benjamin, Inc., New York-Amsterdam, 1965. MR0218496 (36:1582)

[3] E. Yu. Panov, On a class of systems of quasilinear conservation laws, Mat. Sb. 188 (1997), no. 5, 85-112; English transl., Sb. Math. 188 (1997), no. 5, 725-751. MR1478631 (98m:35128)

[4] - On a nonlocal theory of generalized entropy solutions of the Cauchy problem for a class of hyperbolic systems of conservation laws, Izv. Ross. Akad. Nauk Ser. Mat. 63 (1999), no. 1, 133-184; English transl., Izv. Math. 63 (1999), no. 1, 129-179. MR1701842 (2000e:35140)

[5] - On the symmetrizability of first-order hyperbolic systems, Dokl. Akad. Nauk 396 (2004), no. 1, 28-31; English transl. in Dokl. Math. MR2115906

Novgorod State University, Russia

E-mail address: Eugeny.Panov@novsu.ru

Received 29/JAN/2007

Translated by THE AUTHOR 